\title{
Tuning the properties of graphene using a reversible gas-phase reaction
}

\begin{abstract}
Lin Gan ${ }^{1}$, Jian Zhou ${ }^{2}$, Fen $\mathrm{Ke}^{3}$, Hang Gu${ }^{1}$, Danna $\mathrm{Li}^{1}$, Zonghai $\mathrm{Hu}^{3}$, Qiang Sun ${ }^{2}$ and Xuefeng Guo ${ }^{1,2}$
Graphene, because of its remarkable electronic and structural properties, has attracted considerable attention in both the scientific and technological communities. However, a major roadblock to the realization of graphene-based field-effect transistors is that large-area graphene behaves as a semimetal with zero bandgap, making it unsuitable for real applications in sensing, detecting and switching systems. Surface functionalization could result in the construction of periodic micro/ nanostructures by breaking $s p^{2}$ bonds and forming $s p^{3}$ bonds. Therefore, direct chemical grafting might provide a useful way to covalently modify graphene to tailor its properties. Owing to the inert reactivity of its surface, however, only a few chemical reactions have been used to modify its atomic structure. Here, we demonstrate a controllable and efficient means of mild plasma methylation to manipulate the reversible interconversion of two distinct species of graphene (one crystalline and the other methylated). The strategy of incorporating diverse functional substituents (that is, methyl groups and hydrogen atoms) into graphene, instead of a single type of chemical group, could provide a useful route for the development of different applications, such as chemical/biosensors and multifunctional electrical circuits. Moreover, this finely tunable, methylated graphene is stable at room temperature, which suggests that it has intrinsic potential for novel applications in graphene-based optoelectronic devices, inviting further studies.
\end{abstract}

NPG Asia Materials (2012) 4, e31: doi:10.1038/am.2012.58; published online 23 November 2012

Keywords: electronic device; graphene; plasma methylation; surface functionalization

\section{INTRODUCTION}

Graphene, a two-dimensional (2D) crystalline monolayer made of $s p^{2}$-hybridized carbon atoms arranged in a honeycomb lattice, has attracted considerable attention in both the scientific and technological communities. ${ }^{1-4}$ In particular, many scientists in the electrondevice community have recently been active in graphene research because of its possible applications in many emerging areas such as graphene-based electronic devices. ${ }^{5}$ In fact, graphene has been considered a candidate material for post-silicon electronics, according to the strategic planning report by the International Technology Roadmap for Semiconductors. ${ }^{6}$ However, a major roadblock to the realization of graphene-based field-effect transistors is that large-area graphene behaves as a semimetal with zero bandgap, making it unsuitable for real applications in sensing, detecting and switching systems. Therefore, tuning the properties and the bandgap of graphene in a controlled and practical fashion is the primary challenge facing the scientific community at this time.

Theoretical calculations ${ }^{7-9}$ have suggested that the direct chemical grafting process could result in the formation of covalent bonds on the basal planes of graphene sheets, necessitating the breaking of $s p^{2}$ bonds and the formation of $s p^{3}$ bonds, which are followed by the construction of periodic micro/nanostructures. Therefore, surface functionalization, which has proved useful in enhancing the semiconducting characteristics of single-walled carbon nanotubes, ${ }^{10-13}$ should provide an efficient way to covalently modify graphene and thus tailor its chemical, electronic, and mechanical properties. Among the approaches developed so far, ${ }^{14}$ such as hydrogenation, ${ }^{15-17}$ diazonium chemistry, ${ }^{18-20}$ cycloaddition, ${ }^{21}$ photochemistry, ${ }^{22}$ and fluorination, ${ }^{23}$ gas-phase functionalization techniques would be superior for modulating the electronic properties of $s p^{2}$ carbon nanomaterials because of their ease of control, mild conditions and the high reactivity of plasma species. For example, Elias et al. ${ }^{15}$ successfully demonstrated the complete transformation of graphene from its conductive zero-overlap semimetal state to an insulating state (graphane) through a hydrogenation process. In this study, we provide a controllable and efficient means of asymmetric plasma methylation for modulating the electronic, optical and structural properties of single-layer graphenes (SLGs), as evidenced by electrical characterization, Raman spectroscopy, fourier transform infrared spectrometry, X-ray photoemission spectroscopy, high-resolution scanning tunneling microscopy and transmission electron microscopy (TEM). With

${ }^{1}$ Center for NanoChemistry, Beijing National Laboratory for Molecular Sciences (BNLMS), State Key Laboratory for Structural Chemistry of Unstable and Stable Species, College of Chemistry and Molecular Engineering, Peking University, Beijing, China; ${ }^{2}$ Department of Materials Science and Engineering, Peking University, Beijing, China and ${ }^{3}$ College of Physics, Peking University, Chenfulu 202, Haidian District, Beijing, China

Correspondence: Professor X Guo, Center for NanoChemistry, Beijing National Laboratory for Molecular Sciences (BNLMS), State Key Laboratory for Structural Chemistry of Unstable and Stable Species, College of Chemistry and Molecular Engineering, Peking University, Chenfulu 202, Haidian District, Beijing 100871, China.

E-mail: guoxf@pku.edu.cn

Received 9 April 2012; revised 10 October 2012; accepted 12 October 2012 
proper plasma powers and exposure durations, the methylated and hydrogenated coverage of SLGs can be manipulated up to the saturation state $(\sim 25 \%)$. This study is different from that by Dai et al., ${ }^{13}$ which showed that plasma methylation followed by an annealing process can selectively etch and gasify metallic nanotubes while retaining the semiconducting nanotubes in their near-pristine form, thus improving the semiconducting characteristics of the resulting devices.

\section{MATERIALS AND METHODS}

\section{Graphene growth and device fabrication}

In this study, we developed a nondestructive method to fabricate graphenebased transistors using a polymer-mediated transfer technique and selective oxygen plasma etching. A schematic representation of the fabrication procedure is given in Supplementary Figure S1. Each step is quite simple, requiring neither traditional photolithography nor complicated electron beam lithography. Within a very short time, this facile process allows us to mass-produce graphene-based transistor arrays. We first grew large-area SLGs on $\mathrm{Cu}$ foils using a chemical vapor deposition process (CVD), according to procedures reported in the literature. ${ }^{24,25}$ During graphene growth, we fixed the $\mathrm{CH}_{4}$ flow at $\sim 1$ s.c.c.m (standard cubic centimeter per minute) and adjusted the $\mathrm{H}_{2}$ flow to keep a growth pressure of $\sim 60 \mathrm{~Pa}$. We found that, with this modification, we could achieve high-quality graphene sheets, as indicated by the suppression of the D peak in Raman spectrum shown in Supplementary Figure S2d. After graphene growth, polymethyl methacrylate (PMMA) with a 300-nm thickness was spin-coated on the $\mathrm{Cu}$ films. The whole samples were then immersed in a continually refreshed, saturated solution of iron nitrate for a long enough time to remove the $\mathrm{Cu}$ substrates. The resultant transparent sheet floating in the aqueous solution was transferred to a target silicon substrate (Supplementary Figure S2a). To form graphene sheets, we directly applied an oxygen plasma etching process (oxygen: $19 \mathrm{~cm}^{3} \cdot \mathrm{min}^{-1}$, pressure: $35 \mathrm{~Pa}$, power: $50 \mathrm{~W}$ ) for $2.5 \mathrm{~min}$, without removing the PMMA thin films after the transfer step, to etch away the unprotected graphene beneath the exposed PMMA through a shadow mask. Then, after removing the remaining PMMA in a boiling acetone solution, graphene sheet arrays with the desired patterns were obtained. Finally, we made the transistor arrays through a simple thermal evaporation with the aid of another shadow mask. One key advantage of our device fabrication process is its high yield and the uniform electronic properties of the resulting materials. To show this, we fabricated 200 devices on a single substrate over a large area $(\sim 8 \mathrm{~mm} \times 6 \mathrm{~mm})$. A representative device array is shown in Figures S2b and S2c. Only three of the 200 devices are obviously damaged, and the other devices are all conductive, showing yields as high as $98 \%$. Based on the electronic characterization, we estimate the average sheet resistance to be $\sim 0.7-2.0 \mathrm{k} \Omega$. Supplementary Figure S2d shows a representative Raman spectrum from a random graphene sheet. A single symmetric $2 D$ peak (full width at half-maximum $\sim 34 \mathrm{~cm}^{-1}$ ), a small $G / 2 D$ ratio and a negligible D peak are observed, which strongly suggest that our graphene is a single layer with high quality, also demonstrated by TEM (Supplementary Figure S2e). The high yield of the highly conductive devices demonstrated here and the Raman features confirm the growth of high-quality SLGs over a large area and the efficiency of our nondestructive transfer technique.

\section{Plasma methylation and characterization}

To avoid physical destruction by high-energy methyl plasma, we optimized the plasma power and exposure duration. In the vacuum chamber of the reactive ion etching machine, we carefully applied methyl plasma $\left(\mathrm{CH}_{4}\right.$ : 10 s.c.c.m. pressure: $30 \mathrm{~Pa}$, power: $60 \mathrm{~W}$ ) to initiate the surface functionalization of the SLGs at room temperature. Under the optimized conditions, we found that it typically required $30 \mathrm{~min}$ of plasma treatment to reach the saturation state.

The Raman spectra were recorded from SLGs made by a mechanical exfoliation technique using a Horiba HR800 Raman system with $514.5 \mathrm{~nm}$ line from an $\mathrm{Ar}+$ laser (Spectra-Physics model 163-C4205). The X-ray photoemission spectroscopy measurements were carried out on CVD-grown SLGs on silicon wafers with a 300-nm-thick $\mathrm{SiO}_{2}$ layer using a Kratos Analytical Ltd Axis Ultra Imaging Photoelectron Spectrometer (Kyoto, Japan) with $\mathrm{Al} \mathrm{Ka}$ radiation $(225 \mathrm{~W}, 15 \mathrm{~mA}, 15 \mathrm{kV})$. The infrared spectrometry spectra were recorded from CVD-grown SLGs on $100 \mathrm{~nm} \mathrm{SiO}{ }_{2}$ silicon wafers using a Nicolet Magna-infrared spectrometry 750 Fourier transform infrared spectrometer (Nicolet Ltd, Madison, WI, USA). The scanning tunneling microscopy experiments were performed in a standard ultra-high vacuum chamber with a base pressure of $5 \times 10^{-11} \mathrm{mbar}$ on copper substrates used for graphene growth at a bias voltage of $-320 \mathrm{mV}$. The TEM electron diffraction experiments were carried out on CVD-grown SLGs transferred to TEM grids using a field-emission electron microscope (Tecnai F20, FEI, Hillsboro, OR, USA) equipped with a digital camera operated at an accelerating voltage of $200 \mathrm{kV}$. The whole samples were $50-100 \mu \mathrm{m}$, with a selected area $0.4 \mu \mathrm{m}$ in diameter for the electron diffraction experiment. The devices were characterized using a standard probe station and semiconducting parameter analyzer (Agilent 4155C, Agilent Technologies Co., Ltd, Santa Clara, CA, USA). All reactions and measurements were performed at room temperature.

\section{RESULTS AND DISCUSSION}

\section{Electronic properties of graphene after plasma treatment}

To electrically follow the reactions, we developed a facile and nondestructive method to fabricate graphene-based transistor arrays through a polymer-mediated transfer technique and selective oxygen plasma etching. Using neither traditional photolithography nor complicated electron beam lithography, one can mass-produce high-quality graphene-based transistors in high yield within a short time (see Materials and Methods). For graphene growth, we used a CVD method to form large-area SLGs with high quality on $\mathrm{Cu}$ foils, ${ }^{24,25}$ as demonstrated by Raman spectroscopy (Supplementary Figure S2d), TEM images (Supplementary Figure S2e) and TEM electron diffraction (Supplementary Figure S3). After device fabrication, all transferred graphene samples $(\sim 70 \mu \mathrm{m}$ wide $)$ were annealed up to $400{ }^{\circ} \mathrm{C}$ in an argon atmosphere for $4 \mathrm{~h}$ to further remove any possible residues. To minimize any physical damage by high-energy ions, we carefully optimized the plasma power and exposure duration. Under the optimized conditions $\left(\mathrm{CH}_{4}: 10\right.$ s.c.c.m., pressure: $30 \mathrm{~Pa}$, power: $60 \mathrm{~W}$ ), we found that the reaction typically required $30 \mathrm{~min}$ of plasma treatment to reach the saturation state in electrical and Raman characteristics. Figure $1 \mathrm{a}$ and $\mathrm{b}$ illustrate the effect of plasma treatment on the electrical properties of SLG devices (Figure 1a, inset). The extracted carrier mobility of this device is moderate, $\sim 500 \mathrm{~cm}^{2} \mathrm{~V}^{-1} \mathrm{~s}^{-1}$, which could be enhanced by growing largedomain single-crystalline graphene and/or improving the surface cleanness. Before the reaction, the as-prepared SLG transistors exhibited p-type electric field effects with little gate dependence because the neutrality point $\left(I_{\mathrm{NP}}\right)$ shifted to the more-positive value (black curve in Figure 1a), most likely due to chemical doping by etching agents and polymer resists (iron nitrate and PMMA), which could have occurred during graphene transfer and device fabrication. ${ }^{25,26}$ Interestingly, after $30 \mathrm{~min}$ of plasma treatment, dramatic drops in the drain current $\left(I_{\mathrm{D}}\right)$ of these devices were observed (red curve in Figure 1a). As methane plasma produces methyl and hydrogen ion pairs, the current drop can be explained by the covalent attachment of methyl groups and hydrogen atoms to the basal plane of SLGs (Figure 1d), leading to the transition of the graphene carbon atoms from $s p^{2}$ to $s p^{3}$ hybridization (and, therefore, a decrease in the device conductance). Two additional important features should be noted. One is that the neutrality point $\left(I_{\mathrm{NP}}\right)$ of the devices after plasma treatment consistently became more negative $(\sim 20 \mathrm{~V})$, most likely due to the doping effect of electron-donating methyl groups. ${ }^{7}$ The other important feature is that, after plasma treatment, these devices exhibited obviously improved gate dependences. For example, the on/off ratio of the device shown in Figure 1a increased from $<2$ to above 6 . In combination with the aforementioned current drop, the 
a
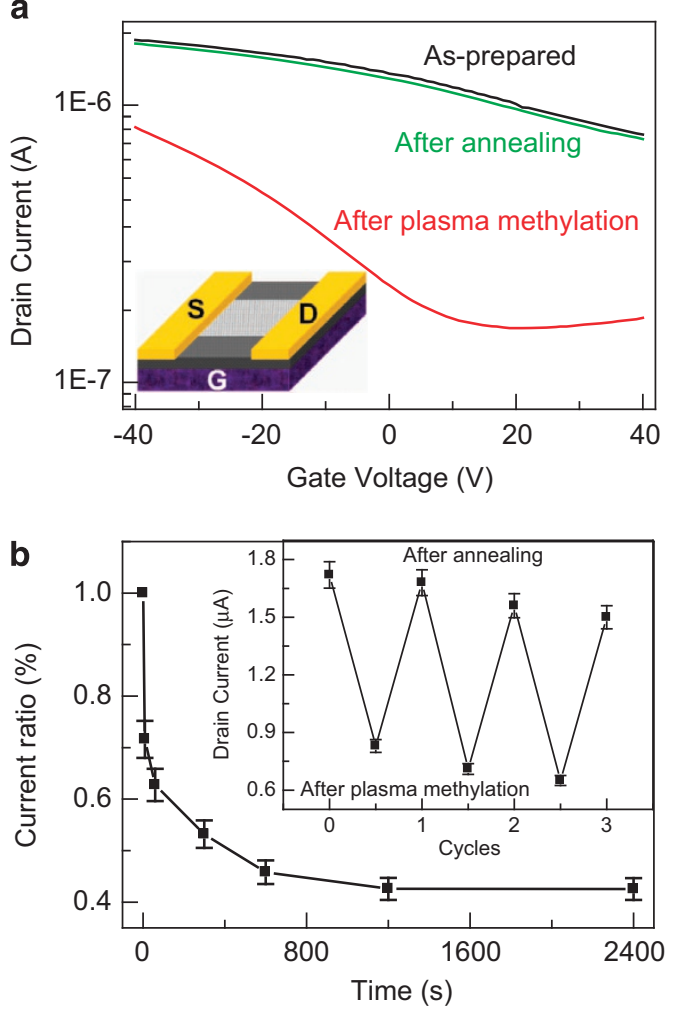

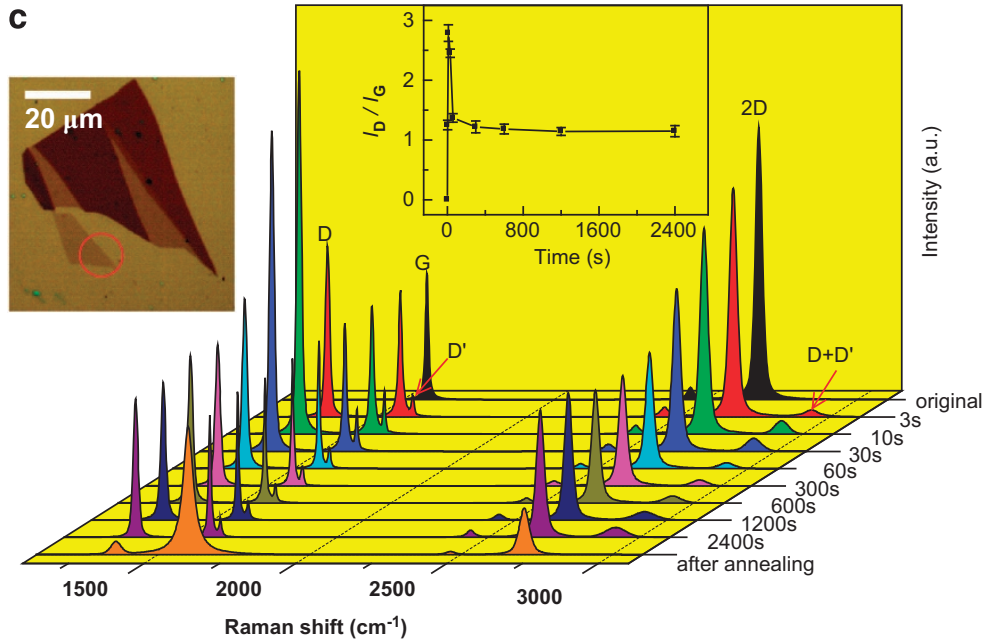

d

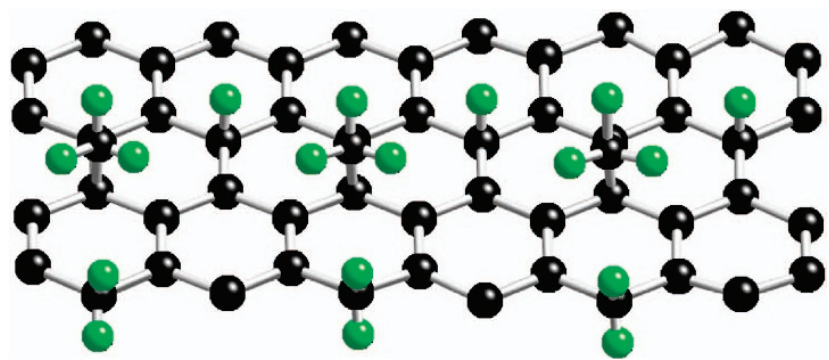

Figure 1 Electrical and Raman characterizations of graphenes before and after plasma treatment. (a) Device characteristics of a representative graphene transistor before plasma treatment, after plasma treatment and after annealing. The inset shows the device structure. (b) The time-evolution of drain currents from the same device during plasma exposure. The inset shows the switching cycles of the same device under the sequential plasma and annealing treatments. (c) Time-dependent changes in the Raman spectra of a single-layer graphene (SLG) caused by plasma methylation. The inset pictures show an optical image of a SLG with the area used for the Raman measurement marked with a red circle and the dependence of the $I_{D} / I_{G}$ ratios versus reaction time for the same SLG, respectively. (d) A schematic of the possible chemical groups on the SLGs after plasma treatment. The black and green spheres represent carbon and hydrogen atoms, respectively.

shift of $I_{\mathrm{NP}}$ and the improved gate modulation suggest that chemical grafting of various functional groups could be a promising strategy for modulating doping-like effects and tuning the electronic properties of graphene devices. ${ }^{7-9}$ We found that plasma methylation on graphene surfaces was rather gradual over time. Figure $1 \mathrm{~b}$ shows the time-evolution of the drain currents $\left(I_{\mathrm{D}}\right)$ of the same device during the plasma exposure. The reaction proceeded reasonably fast in a controllable manner, as $I_{\mathrm{D}}$ decreased to the saturation state within the first $20 \mathrm{~min}$ of reaction. The rate of the methylation reaction became slower and slower with an increasing reaction time, most likely because of the decreased number of active reaction sites and the steric effect of methyl groups that have already been attached to the surface.

\section{Optical properties of graphene after plasma treatment}

The surface methylation of SLGs has been corroborated using Raman spectroscopy. To avoid the influence of any possible residues (for example, photoresist), we used pristine SLGs made by a mechanical exfoliation technique for the Raman characterization. Figure 1c shows the time-evolution of the Raman spectra of a representative SLG sample (Figure 1c, inset) during the plasma exposure. Before plasma treatment, we observed the characteristic peaks in the Raman spectrum of graphenes at $\sim 1580$ and $2680 \mathrm{~cm}^{-1}$, which are ascribed to $\mathrm{G}$ and $2 \mathrm{D}$ bands, respectively. The $\mathrm{G}$ peak corresponds to optical $E_{2 \mathrm{~g}}$ phonons at the Brillouin zone center, whereas the 2D band is the overtone of the $\mathrm{D}$ band usually observed at $\sim 1340 \mathrm{~cm}^{-1}$, which is indicative of the presence of defects and serves as a convenient measure for the amount of disorder in graphenes. ${ }^{27,28}$ After plasma treatment, three new peaks at $\sim 1340,1620$ and $2920 \mathrm{~cm}^{-1}$ were observed, which should be assigned to $\mathrm{D}, \mathrm{D}^{\prime}$ and $\mathrm{D}+\mathrm{D}^{\prime}$ bands, respectively. The D peak at $1340 \mathrm{~cm}^{-1}$ is very sharp compared with that in disordered or nanostructured carbon-based materials, with a full width at half-maximum of $\sim 18 \mathrm{~cm}^{-1}$. $^{28}$ The $\mathrm{D}^{\prime}$ peak at $1620 \mathrm{~cm}^{-1}$, which occurs via an intravalley double-resonance Raman process only in the present of defects, is rather weak and tends to merge into the $\mathrm{G}$ band as a shoulder. The weak $\mathrm{D}+\mathrm{D}^{\prime}$ peak at $2920 \mathrm{~cm}^{-1}$ is a combination of the $\mathrm{D}$ and $\mathrm{D}^{\prime}$ modes. ${ }^{28}$ Unlike the $2 \mathrm{D}$ and $\mathrm{D}^{\prime}$ bands, this band requires a defect for its activation because it is a combination of two phonons with different momentum. Therefore, the observation of $\mathrm{D}, \mathrm{D}^{\prime}$ and $\mathrm{D}+\mathrm{D}^{\prime}$ bands proves the presence of defects in the graphene lattice, which were likely induced by plasma methylation, resulting in the breaking of the translational symmetry of $\mathrm{C}=\mathrm{C} s p^{2}$ bonds and the formation of $\mathrm{C}-\mathrm{C}$ or $\mathrm{C}-\mathrm{H} s p^{3}$ bonds (Figure 1d).

In addition to the observed appearance of new peaks, we also noticed a slight broadening and a decrease in the intensity of the $2 \mathrm{D}$ band relative to the $G$ band consistent with those found in hydrogenated graphenes, ${ }^{15}$ most likely due to chemical doping induced by plasma methylation. ${ }^{29}$ Interestingly, we found that the $I_{\mathrm{D}} / I_{\mathrm{G}}$ ratio has a nonmonotonic dependence on time. The evolution of the resulting $I_{\mathrm{D}} / I_{\mathrm{G}}$ data as a function of time is shown in Figure 1c, inset. Within the first 10 seconds of plasma exposure, $I_{\mathrm{D}} / I_{\mathrm{G}}$ ratios 

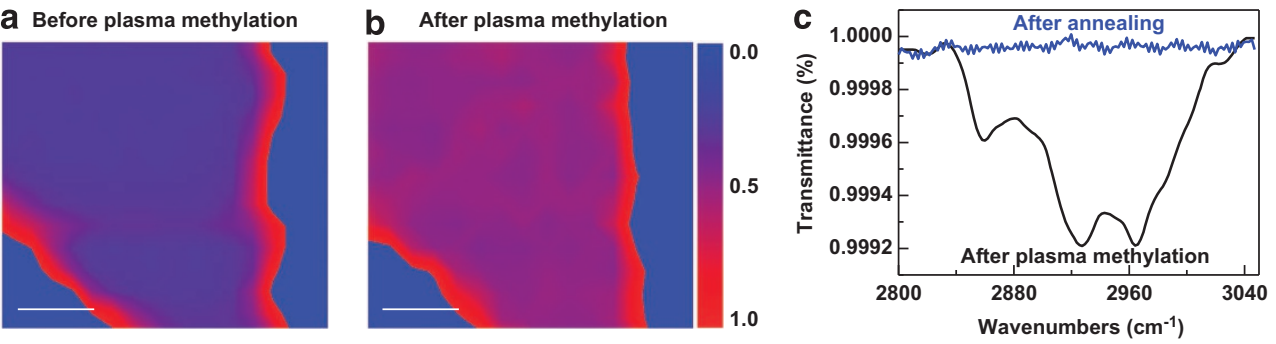

Figure 2 Comparison of graphenes before and after plasma methylation via two-dimensionalRaman spectra and fourier transform infrared spectrometry studies. (a and b) Spatial maps of the $I_{D} / I_{G}$ ratios over the circled area of the same SLG used in Figure 1c before (a) and after plasma methylation (b). The scale bar is $1 \mu \mathrm{m}$. (c) Infrared transmittance spectra of a single-layer graphene (SLG) after plasma treatment and further annealing.

sharply increased to a peak value and then gradually decreased to the saturation level. Such a behavior can be explained by the existence of two disorder-induced, competing mechanisms contributing to the D band. In the first stage, a proper quantification of the amount of defects in graphene has been given by the well-known TuinstraKoenig relation, ${ }^{30} L_{\mathrm{a}}=\left(2.4 \times 10^{-10}\right) \lambda^{4}\left(I_{D} / I_{G}\right)^{-1}$, where $L_{\mathrm{a}}$ is the size of in-plane crystallites formed by a certain number of carbon rings and $\lambda$ is the laser excitation wavelength. It can be assumed that a higher number of defects with increasing plasma treatment results in higher D peak intensity and a lower $L_{\mathrm{a}}$. However, in the second stage, the Tuinstra-Koenig relation was broken down according to $\left(I_{\mathrm{D}} / I_{\mathrm{G}}\right)$ $\propto\left(L_{\mathrm{a}}^{2}\right)$. This result provides the experimental support for a phenomenological model proposed by Lucchese et al. ${ }^{31}$ which could be useful for quantifying disorder in graphene monolayers.

We found that the plasma-treated SLG samples were quite stable, as they retained their electrical and Raman characteristics when kept in the dark for at least two weeks at room temperature. Interestingly, after annealing at $400{ }^{\circ} \mathrm{C}$ under an $\mathrm{Ar}$ atmosphere for $\sim 2 \mathrm{~h}$, we were able to restore the original electrical and Raman properties of the samples. After annealing, the device characteristics of the functionalized devices almost recovered to their initial value and shape (green curve in Figure 1a). The sequential steps of plasma treatment and the annealing process can be operated in a reversible manner for at least three switching cycles while keeping the devices in good shape (Figure $1 \mathrm{~b}$, inset). The recovery was not complete, however, which might be due to vacancies induced by plasma damage and/or residual oxygen during oven annealing. ${ }^{15,32}$ Consistently, all of the defectrelated peaks in the Raman spectrum $\left(\mathrm{D}, \mathrm{D}^{\prime}\right.$ and $\left.\mathrm{D}+\mathrm{D}^{\prime}\right)$ completely disappeared or were strongly suppressed after annealing (Figure 1c). However, the residual D and broadened G peaks were still observed, indicating the presence of some disorder in the annealed graphene. These changes in the Raman spectra are in good agreement with the electrical measurements.

To probe the reaction extent, we screened the spatial maps of the $I_{\mathrm{D}} / I_{\mathrm{G}}$ ratios over the circled area of the same SLG used in Figure 1c before and after plasma methylation. As shown in Figures $2 \mathrm{a}$ and $\mathrm{b}$, the whole region exhibits both large-area uniformity and a much greater degree of disorder after methylation than before methylation. The intensity of the defect $\mathrm{D}$ band is as great as $\sim 120 \%$ of that of the $\mathrm{G}$ band, which is consistent with the previous $I_{\mathrm{D}} / I_{\mathrm{G}}$ ratio measurements. These results suggest that a periodic patterning of methyl groups and hydrogen atoms on the graphene surfaces at the saturation state may have formed, which could result in a confinement potential for the carriers in graphene and potentially lead to a bandgap opening. ${ }^{33}$

To check the chemical effects of plasma methylation, we carried out fourier transform infrared spectrometry experiments. After plasma treatment, we detected infrared spectrometry vibrational modes at $\sim 2964,2927$ and $2857 \mathrm{~cm}^{-1}$ (black curve in Figure 2c), which should be assigned to $\mathrm{C}-\mathrm{CH}_{3}, \mathrm{C}-\mathrm{H}$ or $\mathrm{C}-\mathrm{H}_{2}$ and $\mathrm{C}-\mathrm{H}_{2}$, respectively (Figure 1d). This observation is in perfect agreement with those in Dai's work, ${ }^{13}$ where methyl groups and hydrogen atoms can be grafted on carbon nanotube sidewalls through a gas-phase reaction. After annealing, these peaks completely vanished, indicating the elimination of covalently bound groups through demethylation and dehydrogenation. These results are consistent with those obtained above in the electrical and Raman characterizations.

\section{Structural properties of graphene after plasma treatment}

To obtain a deeper understanding of the chemical properties of the methylated graphenes, X-ray photoemission spectroscopy studies were carefully performed. Figure $3 \mathrm{a}$ and $\mathrm{b}$ show comparisons of the representative C 1s core-level spectra of the same SLG sample before and after plasma treatment. Generally, the C 1s spectra were fitted with four components before plasma treatment. The main peak at $284.8 \mathrm{eV}$ is assigned to the C-C $s p^{2}$ bonds in the graphene hexatomic ring structure, and the other three peaks with higher-binding energies at 286.0, 286.8 and $289.1 \mathrm{eV}$ should be assigned to $\mathrm{C}-\mathrm{OH}, \mathrm{C}=\mathrm{O}$ and $\mathrm{C}(\mathrm{O}) \mathrm{O}$ bonds, respectively, and may originate from oxidization, from iron nitrate and/or $\mathrm{O}_{2}$ in air. The peak of the trace C-N bonds, which might result from copper etching by iron nitrate (Supplementary Figure S4), is overlaid with the peak of the $\mathrm{C}=\mathrm{O}$ bonds. ${ }^{34,35}$ After plasma treatment, we found that the $\mathrm{C} 1 \mathrm{~s}$ peak became much simpler. The main peak at $284.8 \mathrm{eV}$ still corresponded to $s p^{2}$-hybridized C atoms in graphene. However, compared with the results before plasma treatment, two significant differences should be highlighted. One is that a prominent peak is newly formed at $285.4 \mathrm{eV}$, which should be assigned to the $s p^{3}$ hybridized $\mathrm{C}$ atoms. ${ }^{36}$ This observation strongly strengthened the fact that plasma methylation results in the breaking of the translational symmetry of $\mathrm{C}=\mathrm{C} s p^{2}$ bonds and the formation of $\mathrm{C}-\mathrm{C}$ or $\mathrm{C}-\mathrm{H} s p^{3}$ bonds. The other is that the other three peaks (that is, at $286.0 \mathrm{eV}(\mathrm{C}-\mathrm{OH}), 286.8 \mathrm{eV}(\mathrm{C}=\mathrm{O}$ and/or $\mathrm{C}-\mathrm{N})$ and $289.1 \mathrm{eV}$ $(\mathrm{C}(\mathrm{O}) \mathrm{O})$ ) completely vanished after plasma treatment. This is consistent with the results from the whole X-ray photoemission spectroscopy spectra (Supplementary Figure S4), where we found that the amounts of $\mathrm{O}$ and $\mathrm{N}$ atoms were strongly suppressed after plasma treatment. We speculate that, based on the above observations, methane plasma ignites the reaction at a defect on the graphene surface (either present before plasma treatment or induced by the plasma) and then continues to react with graphene. From the data in Figure $3 \mathrm{~b}$, the ratio of the integrated areas for $s p^{3}$ - and $s p^{2}$-hybridized carbon components is $\sim 0.51$. As methane plasma produces methyl and hydrogen ion pairs, if we hypothesize that both of the active species can simultaneously react with graphene to form $\mathrm{C}-\mathrm{CH}_{3}$ and 

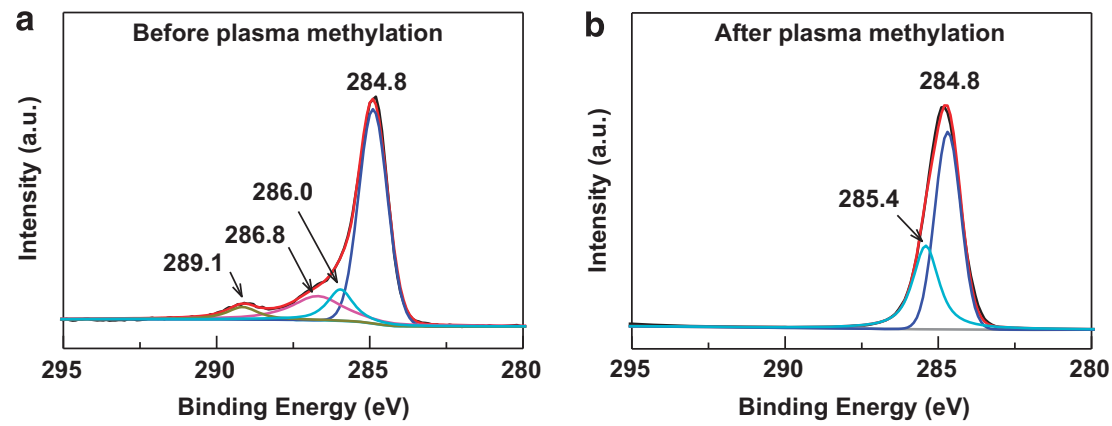

Figure 3 X-ray photoemission spectroscopy (XPS) studies of methylated graphenes. The C $1 \mathrm{~s}$ XPS spectra of the same single-layer graphene (SLG) sample before (a) and after plasma treatment for $30 \mathrm{~min}(\mathbf{b})$.
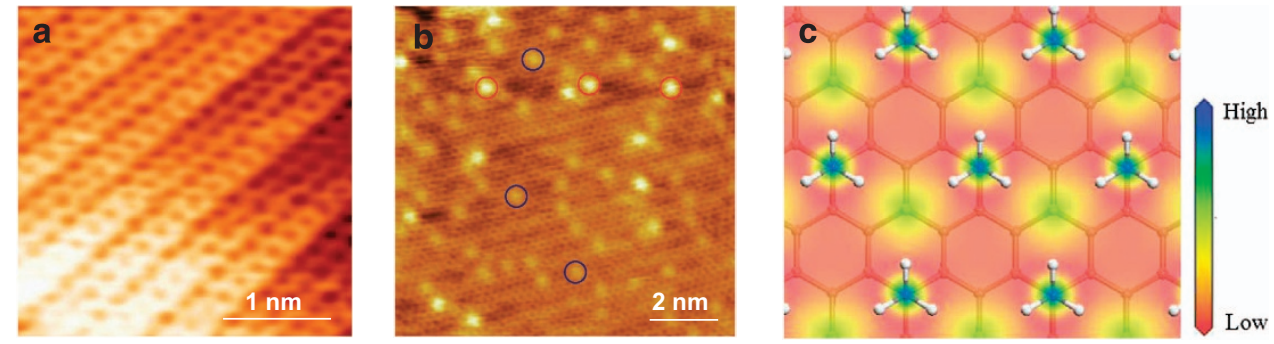

Figure 4 Scanning tunneling microscopy studies of methylated graphenes. High-resolution scanning tunneling microscopy images of the same single-layer graphene sample before (a) and after plasma methylation (b). After plasma methylation, two types of distinct quantum dots with different levels of brightness (labeled with red and blue circles) were observed at a bias voltage of $-320 \mathrm{mV}$. (c) Simulated scanning tunneling microscopy image of the model under $-0.3 \mathrm{~V}$ bias voltage, showing that methyl groups are brighter than hydrogen atoms.
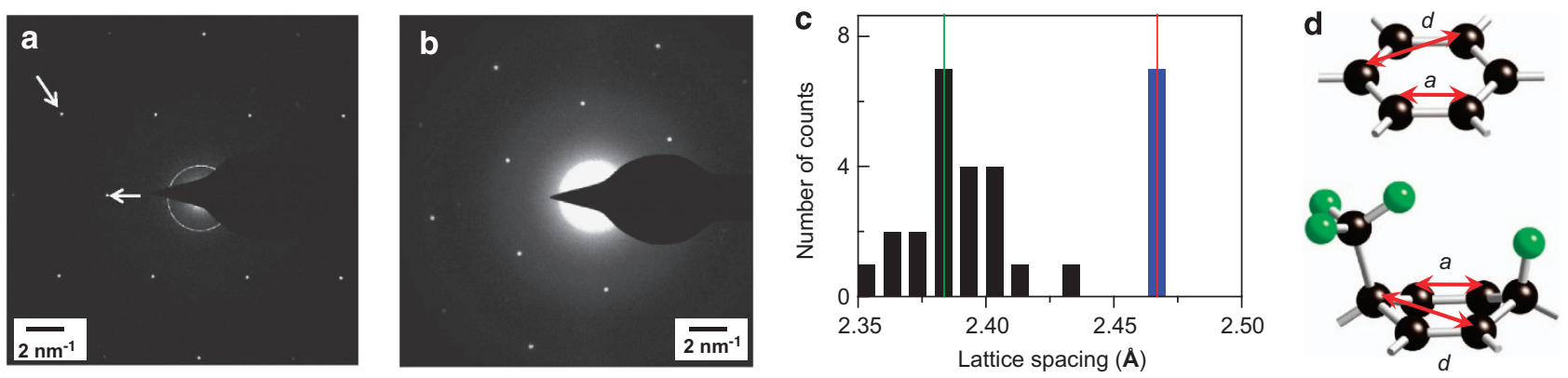

Figure 5 Transmission electron microscopy (TEM) studies of methylated graphenes. TEM electron diffraction patterns of the same single-layer graphene sample before (a) and after plasma treatment for $30 \mathrm{~min}$ (b). The intensity of the diffraction spots indicated by the arrows in Figure 5 a from the outer hexagon is equivalent to that from the inner hexagon, confirming that our graphene samples are single-layer (Supplementary Figure S3). (c) Distribution of the lattice distance $d$ in methylated graphenes (black). The green line marks the average value after plasma treatment, whereas the red line shows the $d$ observed in graphene (both before plasma treatment and after annealing). (d) Schematic representation of the possible crystal structure of graphene (top) and methylated graphene (bottom). The black and green spheres represent carbon and hydrogen atoms, respectively.

$\mathrm{C}-\mathrm{H}$ bonds, respectively, ${ }^{13}$ we can calculate the maximum defect coverage on graphene surfaces to be $\sim(0.51 \times 2 / 3) /(0.51 \times 2 /$ $3+1)=\sim 25 \%$, where $100 \%$ defect coverage indicates that each carbon atom in the basal plane of graphene was methylated or hydrogenated. This value is consistent with the theoretical prediction ( $25 \%$ coverage) that could form the electronic structure with the most stable configuration, possibly resulting in a quantum confinement for a bandgap opening. ${ }^{7}$ Atomic force microscopic characterizations showed that the average thickness increase of SLGs after plasma methylation was $\sim 0.21 \mathrm{~nm}$ (Supplementary Figure S5). By controlling the reaction extent, we were able to successfully see the individual absorbed atoms or groups using high-resolution scanning tunneling microscopy. Before plasma treatment, a clean, densely packed honeycomb crystal lattice of pristine graphenes was observed (Figure $4 \mathrm{a})$. Figure $4 \mathrm{~b}$ shows a representative scanning tunneling microscopy image of graphene after plasma treatment with a reaction coverage of $\sim 5 \%$. Interestingly, we found two types of distinct quantum dots with different levels of brightness, which should be ascribed to methyl groups (bright with the red circle) and hydrogen atoms (dark with the blue circle), respectively. This observation is well explained by the theoretical simulations under $-0.3 \mathrm{~V}$ bias voltages shown in Figure 4c, which show that methyl groups are brighter than hydrogen atoms (more details about the theoretical calculations can be found in the Supplementary Information). It is the use of methyl plasma that allows us to flexibly control the reaction extent and graphene's atomic structure (and potentially its band structure). 
More structural information about methylated SLG samples are provided by TEM studies. Figures $5 \mathrm{a}$ and $\mathrm{b}$ show a comparison of the representative TEM electron diffraction patterns from dozens of measured samples. Again, the intensity of the diffraction spots from the outer hexagon being almost equivalent to the intensity from the inner hexagon confirms that our graphene samples are single layer (Supplementary Figure S3). ${ }^{27}$ Before plasma treatment, all samples consistently exhibited clear and strong diffraction patterns (Figure $5 \mathrm{a}$ ), indicating crystalline hexagonal symmetry with a narrow lattice constant $d(2.46 \pm 0.02 \AA)$. In contrast, plasma methylation led to a dramatic change in the lattice constant $d$ but preserved the hexagonal symmetry and corresponding crystallinity, so the diffraction images became obscure (Figure $5 \mathrm{~b}$ ). In real experiments, the changes in the lattice constant $d$ were not uniform. As shown in Figure $5 c$, another area of the methylated graphene exhibited locally different in-plane periodicities with the average decrease being $\sim 3.3 \%$. Such a nonuniformity is reasonable because the compression force induced by chemical reactions is too tiny to equilibrate the local physical environment of the sample, thus restricting the isotropic shrinkage. As proposed by Elias et al., ${ }^{15}$ the in-plane shrinkage of the graphene lattice should be only the result of chemical modification. Generally, the C-C bond distance $a$ in graphene is $\sim 1.42 \AA$. However, methylation and/or hydrogenation results in the hybridization of carbon atoms from $s p^{2}$ to $s p^{3}$ (Figure $5 \mathrm{~d})$, thus leading to the elongation of C-C bonds $(\sim 1.52 \AA$, close to that of diamond) and the in-plane periodicity $d$ to be $\sim 1 \%$ less than in graphene, consistent with the theoretical calculations discussed in the Supplementary Information. From the experimental distribution of $d$, the dominant value is found at $\sim 2.38 \AA$, suggesting that either shorter or stronger C-C bonds, or both, are present. More sophisticated experimental investigations need to be performed to understand the intrinsic structure of the novel species. Interestingly, annealing treatment led to the complete recovery of the original periodicity of graphene observed in TEM electron diffraction.

\section{CONCLUSIONS}

The combined experimental and theoretical results prove that plasma methylation results in the interconversion of two distinct species of graphene (one crystalline and the other methylated) in a controllable and reversible manner, which induces pronounced changes in the electronic, optical and structural properties of graphene. The methylated graphene is finely tunable and stable at room temperature, suggesting its wide application in electronics. Therefore, these results demonstrate the potential to realize confinement-induced gap opening by sophisticated chemical modification, which might provide appealing opportunities for novel scientific and technological research and innovation with graphene-based optoelectronic devices.

\section{CONFLICT OF INTEREST}

The authors declare no conflict of interest.

\section{ACKNOWLEDGEMENTS}

We thank Zhongfan Liu, Yanfeng Zhang, Teng Gao, Yabo Gao, Rui Zhang and Xiaosong Wu from Peking University for sample preparations and Junfeng $\mathrm{He}$, Zhuojin Xie and Xinjiang Zhou from the Institute of Physics, Chinese Academy of Sciences for helpful discussions. We acknowledge primary financial support from MOST (2009CB623703 and 2012CB921404), NSFC (20833001, 21225311, 51121091, 2112016, and 21003002), FANEDD (2007B21), 111 Project (B08001) and BSTSP (2009A01).
1 Geim, A. K. \& Novoselov, K. S. The rise of graphene. Nat. Mater. 6, 183-191 (2007)

2 Geim, A. K. Graphene Status and prospects. Science 324, 1530-1534 (2009).

3 Castro Neto, A. H., Guinea, F., Peres, N. M. R., Novoselov, K. S. \& Geim, A. K. The electronic properties of graphene. Rev. Mod. Phys. 81, 109-162 (2009).

4 Rao, C. N. R., Sood, A. K., Subrahmanyam, K. S. \& Govindaraj, A. Graphene: the new two-dimensional nanomaterial. Angew. Chem. Int. Ed. 48, 7752-7777 (2009).

5 Schwierz, F. Graphene transistors. Nat. Nanotechnol 5, 487-496 (2010).

6 The International Technology Roadmap for Semiconductors http://www.itrs. net/Links/ 2009ITRS/Home2009.htm Semiconductor Industry Association (2009).

7 Boukhvalov, D. W. \& Katsnelson, M. I. Tuning the gap in bilayer graphene using chemical functionalizatioin: density functional calculations. Phys. Rev. B 78, 085413 (2008)

8 Boukhvalov, D. W., Katsnelson, M. I. \& Lichtenstein, A. I. Hydrogen on graphene: electronic structure, total energy, structural distortions and magnetism from firstprinciples calculations. Phys. Rev. B 77, 035427 (2008).

9 Sofo, J. O., Chaudhari, A. S. \& Barber, G. D. Graphane: a two-dimensional hydrocarbon. Phys. Rev. B 75, 153401 (2007).

10 Zheng, G., Li, Q., Jiang, K., Zhang, X., Chen, J., Ren, Z. \& Fan, S. Transition of single walled carbon nanotubes from metallic to semiconducting in field-effect transistors by hydrogen plasma treatment. Nano Lett. 7, 1622-1625 (2007).

11 Zhang, G. Y., Qi, P., Wang, X., Lu, Y., Mann, D., Li, X. \& Dai, H. Hydrogenation and hydrocarbonation and etching of single-walled carbon nanotubes. J. Am. Chem. Soc 128, 6026-6027 (2006).

12 Kanungo, M., Lu, H., Malliaras, G. G. \& Blanchet, G. B. Suppression of metallic conductivity of single-walled carbon nanotubes by cycloaddition reactions. Science 323, 234-237 (2009).

13 Zhang, G. Y., Qi, P., Wang, X., Lu, Y., Li, X., Tu, R., Bangsaruntip, S., Mann, D., Zhang, L. \& Dai, H. Selective etching of metallic carbon nanotubes by gas-phase reaction. Science 314, 974-976 (2006).

14 Loh, K. P., Bao, Q. L., Ang, P. K. \& Yang, J. X. The chemistry of graphene. J. Mater. Chem. 20, 2277-2289 (2010).

15 Elias, D. C., Nair, R. R., Mohiuddin, T. M. G., Morozov, S. V., Blake, P., Halsall, M. P. Ferrari, A. C., Boukhvalov, D. W., Katsnelson, M. I., Geim, A. K. \& Novoselov, K. S. Control of graphene's properties by reversible hydrogenation: evidence for graphane. Science 323, 610-613 (2009).

16 Balog, R., Jørgensen, B., Nilsson, L., Andersen, M., Rienks, E., Bianchi, M., Fanetti, M., Lægsgaard, E., Baraldi, A., Lizzit, S., Sljivancanin, Z., Besenbacher, F., Hammer, B., Pedersen, T. G., Hofmann, P. \& Hornekær, L. Bandgap opening in graphene induced by patterned hydrogen adsorption. Nat. Mater. 9, 315-319 (2010).

17 Subrahmanyam, K. S., Kumar, P., Maitra, U., Govindaraj, A., Hembram, K. P. S. S., Waghmare, U. V. \& Rao, C. N. R. Chemical storage of hydrogen in few-layer graphene. Proc. Natl. Acad. Sci. USA 108, 2674-2677 (2011).

18 Sinitskii, A., Dimiev, A., Corley, D. A., Fursina, A. A., Kosynkin, D. V. \& Tour, J. M. Kinetics of diazonium functionalization of chemically converted graphene nanoribbons. ACS Nano 4, 1949-1954 (2010).

19 Bekyarova, E., Itkis, M. E., Ramesh, P., Berger, C., Sprinkle, M., de Heer, W. A. \& Haddon, R. C. Chemical modification of epitaxial graphene: spontaneous grafting of aryl groups. J. Am. Chem. Soc. 131, 1336-1337 (2009).

20 Farmer, D. B., Golizadeh-Mojarad, R., Perebeinos, V., Lin, Y.-M., Tulevshi, G. S., Tsang, J. C. \& Avouris, P. Chemical doping and electron-hole conduction asymmetry in graphene devices. Nano Lett. 9, 388-392 (2009).

21 Georgakilas, V., Bourlinos, A. B., Zboril, R., Steriotis, T. A., Dallas, P., Stubos, A. K. \& Trapalis, C. Organic functionalisation of graphenes. Chem. Commun. 46, 1766-1768 (2010).

22 Liu, H. T., Ryu, S., Chen, Z., Steigerwald, M. L., Nuckolls, C. \& Brus, L. E. Photochemical reactivity of graphene. J. Am. Chem. Soc. 131, 17099-17100 (2009).

23 Robinson, J. T., Burgess, J. S., Junkermeier, C. E., Badescu, S. C., Reinecke, T. L., Keith Perkins, F., Zalalutdniov, M. K., Baldwin, J. W., Culbertson, J. C., Sheehan, P. E. \& Snow, E. S. Properties of fluorinated graphene films. Nano Lett. 10, 3001-3005 (2010).

24 Li, X. S., Cai, W., An, J., Kim, S., Nah, J., Yang, D., Piner, R., Velamakanni, A., Jung, I., Tutuc, E., Banerjee, S. K., Colombo, L. \& Ruoff, R. S. Large-area synthesis of high-quality and uniform graphene films on copper foils. Science 324, 1312-1314 (2009).

25 Gan, L., Liu, S., Li, D., Gu, H., Cao, Y., Shen, Q., Wang, Z., wang, Q. \& Guo, X. Facile fabrication of the crossed nanotube-graphene junctions. Acta Phys.-Chim. Sin 26 1151-1156 (2010)

26 Wang, Q., Guo, X., Cai, Li., Cao, Y., Gan, L., Liu, S., Wang, Z., Zhang, H. \& Li, L. $\mathrm{TiO}_{2}$-decorated graphenes as efficient photoswitches with high oxygen sensitivity. Chem. Sci. 2, 1860-1864 (2011).

27 Ferrari, A. C., Meyer, J. C., Scardaci, V., Casiraghi, C., Lazzeri, M., Mauri, F., Piscanec, S., Jiang, D., Novoselov, K. S., Roth, S. \& Geim, A. K. Raman spectrum of graphene and graphene layers. Phys. Rev. Lett. 97, 187401 (2006).

28 Dresselhaus, M. S., Jorio, A., Hofmann, M., Dresselhaus, G. \& Saito, R. Perspectives on carbon nanotubes and graphene raman spectroscopy. Nano Lett. 10, 751-758 (2010).

29 Das, A., Pisana, S., Chakraborty, B., Piscanec, S., Saha, S. K., Waghmare, U. V. Novoselov, K. S., Krishnamurthy, H. R., Geim, A. K., Ferrari, A. C. \& Sood, A. K Monitoring dopants by Raman scattering in an electrochemically top-gated graphene transistor. Nat. Nanotechnol. 3, 210-215 (2008).

30 Tuinstra, F. \& Koenig, J. L. Raman spectrum of graphite. J. Chem. Phys. 53 1126-1131 (1970) 
31 Lucchese, M. M., Stavale, F., Martins Ferreira, E. H., Vilani, C., Moutinho, M. V. O., Capaz, R. B., Achete, C. A. \& Jorio, A. Quantifying ion-induced defects and Raman relaxation length in graphene. Carbon 48, 1592-1597 (2010).

32 Ryu, S., Han, M. Y., Maultzsch, J., Heinz, T. F., Kim, P., Steigerwald, M. L. \& Brus, L. E. Reversible basal plane hydrogenation of graphene. Nano Lett. 8, 4597-4602 (2008).

33 Pedersen, T. G., Flindt, C., Pedersen, J., Mortensen, N. A., Jauho, A.-P. \& Pedersen, K. Graphene antidot lattices: designed defects and spin qubits. Phys. Rev. Lett. 100, 136804 (2008).

34 Malitesta, C., Losito, I., Sabbatini, L. \& Zambonin, P. G. New findings on polypyrrole chemical structure by XPS coupled to chemical derivatization labelling. J. Electron Spectrosc. 76, 629-634 (1995).
35 Niwa, Y., Kobayash, H. \& Tsuchiya, T. X-ray photoelectron-spectroscopy of tetraphenylporphin and phthalocyanine. J. Chem. Phys. 60, 799-807 (1974).

36 Nikitin, A., Naslund, L. A., Zhang, Z. Y. \& Nilsson, A. C-H bond formation at the graphite surface studied with core level spectroscopy. Surf. Sci. 602, 2575-2580 (2008).

\section{(C)}

This work is licensed under the Creative Commons Attribution-NonCommercial-No Derivative Works 3.0 Unported License. To view a copy of this license, visit http://creativecommons.org/licenses/by-nc-nd/3.0/

Supplementary Information accompanies the paper on the NPG Asia Materials website (http://www.nature.com/am) 